\title{
ESTRUTURA POPULACIONAL E MORFOMETRIA DO CAMARÃO BRANCO Litopenaeus schmitti (BURKENROAD, 1936) CAPTURADO PELA PESCA ARTESANAL EM SERGIPE, BRASIL
}

\author{
Matheus Cristóvão de Santana FREIRE ${ }^{1 *}$; Kátia Meirelles Felizola FREIRE ${ }^{1}$; Leonardo Cruz da \\ ROSA$^{2}$; Carolina Nunes Costa BOMFIM ${ }^{3}$
}

\begin{abstract}
1 Departamento de Engenharia de Pesca e Aquicultura/Laboratório de Ecologia Pesqueira, Universidade Federal de Sergipe, 49100-000, São Cristóvão-Sergipe, Brasil. E-mail: matheusfreire17-ufs@outlook.com, kmffreire2018@gmail.com

*Autor correspondente
\end{abstract}

2 Departamento de Engenharia de Pesca e Aquicultura/Laboratório de Ecologia Bentônica, Universidade Federal de Sergipe, 49100-000, São Cristóvão-Sergipe, Brasil. E-mail: leonardo.rosa@rocketmail.com

3 Departamento de Engenharia de Pesca e Aquicultura/Laboratório de Nutrição e Cultivo de Organismos Aquáticos, Universidade Federal de Sergipe, 49100-000, São Cristóvão-Sergipe, Brasil. E-mail: carolncosta@yahoo.com.br

\begin{abstract}
RESUMO. O presente trabalho objetivou analisar a estrutura populacional do camarão branco Litopenaeus schmitti (Burkenroad, 1936) capturado pela pesca artesanal de arrasto em Sergipe e sua morfometria. O material biológico foi coletado mensalmente de janeiro/2015 a março/2016, exceto durante os defesos. De todos os indivíduos foram medidos o comprimento total (CT), o comprimento da carapaça (CC) e o peso total (PT), identificados o sexo e o estágio de maturação. Dos 191 indivíduos coletados, 81 foram machos e 110 fêmeas, resultando em uma proporção sexual de 0,7:1. O comprimento médio da carapaça para os machos foi $26,85 \pm 2,73 \mathrm{~mm}$ CC e para as fêmeas foi $29,97 \pm 5,28 \mathrm{~mm}$ CC. O peso médio foi $16,39 \pm 4,34 \mathrm{~g}$ e $20,55 \pm 9,51 \mathrm{~g}$ para machos e fêmeas, respectivamente. A relação entre o comprimento total e o comprimento da carapaça para machos e fêmeas foi CT=25,960+3,837CC e CT=33,199+3,502CC, respectivamente. A relação entre o peso total e o comprimento da carapaça foi $\mathrm{PT}=0,00303 \cdot \mathrm{CC}^{2,609}$ para machos e PT $=0,00248 \cdot \mathrm{CC}^{2,642}$ para fêmeas. O maior exemplar amostrado foi uma fêmea com $52,50 \mathrm{~mm}$ CC, correspondendo ao maior indivíduo já registrado em Sergipe. Apenas 9,4\% dos exemplares estavam imaturos. Essa é a primeira vez que a estrutura populacional dessa espécie é analisada diretamente na pesca de arrasto artesanal em Sergipe.
\end{abstract}

Palavras-Chave: Pistola, tamanho máximo, análise morfométrica, peneídeo, relação peso-comprimento.

\begin{abstract}
The present study aimed at analyzing the population structure of the southern white shrimp Litopenaeus schmitti (Burkenroad, 1936) caught by the artisanal trawling fishery in Sergipe and its morphometry. Samples were collected monthly from January/2015 to March/2016, except during the closed seasons. Total length (TL), carapace length $(\mathrm{CL})$, and total weight (TW) were measured in all individuals, and sex and stage of maturation were identified. Out of the 191 individuals sampled, 81 were males and 110 females, resulting in a sexual ratio of $0.7: 1$. The mean carapace length for males was $26.85 \pm 2.73 \mathrm{~mm} \mathrm{CL}$ and for females was $29.97 \pm 5.28 \mathrm{~mm} \mathrm{CL}$. The mean weight was $16.39 \pm 4.34 \mathrm{~g}$ and $20.55 \pm 9.51 \mathrm{~g}$ for males and females, respectively. The relationship between total length and carapace length for males and females was $T L=25.960+3.837 \mathrm{CL}$ and $\mathrm{TL}=33.199+3.502 \mathrm{CL}$, respectively. The relationship between total weight and carapace length was $T W=0.00303 \mathrm{CL}^{2.609}$ for males and $\mathrm{TW}=0.00248 \mathrm{CL}^{2.642}$ for females. The largest specimen sampled was a female with $52.50 \mathrm{~mm} \mathrm{CL}$, corresponding to the largest individual ever reported off Sergipe. Only $9.4 \%$ of the specimens were immature. This is the first time that the population structure for this species was directly analyzed using data from artisanal trawling in Sergipe.
\end{abstract}

Keywords: white shrimp, maximum size, morphometric analysis, penaeid, weight-length relationship. 
Freire et al., Estrutura populacional e morfometria do camarão branco Litopenaeus schmitti (Burkenroad, 1936) capturado pela pesca artesanal em Sergipe, Brasil.

\section{INTRODUÇÃO}

A atividade pesqueira marinha e estuarina possui uma grande importância econômica e social para o Brasil, gerando emprego e renda para cerca de quatro milhões de pessoas (Fundação BIO-RIO, 2002). A partir do final da $2^{a}$ Guerra Mundial surgiu uma política de implantação da pesca industrial, fazendo com que a produção da atividade pesqueira marinha brasileira ultrapassasse 700 mil t/ano na metade da década de 1980 (Diegues, 1983). Em 2007, essa produção atingiu 540 mil toneladas (IBAMA, 2007; Vasconcellos et al., 2007). Entre 2013 e 2015, foi obtida uma média anual de 433 mil toneladas (FAO, 2016).

Apesar do vasto litoral e clima adequado para o desenvolvimento de várias espécies, o Brasil, quando comparado com outros países da América do Sul, como o Peru e Chile, p.ex., possui uma baixa produção pesqueira devido ao aporte limitado de nutrientes e ressurgência de pequena magnitude e restrita espacialmente (Flores-Montes, 2003). Entre as espécies de maior importância econômica devido ao seu elevado valor comercial e boa aceitação no mercado estão os camarões marinhos. As famílias de camarões com maior interesse econômico no litoral brasileiro são os Penaeidae, representados principalmente por Xiphopenaeus kroyeri (Heller, 1862), Farfantepenaeus subtilis (Pérez-Farfante, 1967), Farfantepenaeus brasiliensis (Latreille, 1817), Farfantepenaeus paulensis (Pérez-Farfante, 1967) e Litopenaeus schmitti (Burkenroad, 1936), e os Solenoceridae, representados por Pleoticus muelleri (Bate, 1888) (Haimovici et al., 2014). A produção do L. schmitti no Brasil, oriunda da pesca extrativa marinha, foi de cerca de 4100 t em 2007 (IBAMA, 2007). Em Sergipe, a produção total de L. schmitti foi de 157 t em 2013 (Thomé-Souza et al., 2014) e de 135 t em 2014 (Araújo et al., 2016). Apesar de representar uma produção reduzida, seu valor comercial no estado, juntamente com os exemplares de maior porte do camarão rosa, supera o das demais espécies de camarão. Salienta-se que a produção de $L$. schmitti é registrada nos boletins do estado de Sergipe sob o nome comum de camarão branco ou pistola, correspondendo aos camarões de maior porte (Araújo et al., 2016; Thomé-Souza et al., 2014). A categoria de camarão de médio porte, conhecida como escolha, é atribuída a várias espécies de porte médio e o espigão, apenas a $X$. kroyeri (Araújo et al., 2016; Thomé-Souza et al., 2014).

Litopenaeus schmitti distribui-se desde Cuba $\left(23^{\circ} 30^{\prime} \mathrm{N}\right)$ até o estado do Rio Grande do Sul, no Brasil (2945'S) (Pérez-Farfante and Kensley, 1997). Seu ciclo de vida é dependente do estuário, com as pós-larvas chegando às áreas de berçário (estuário) por meio da migração vertical, atingindo a fase juvenil 20 a 30 dias após a desova e, posteriormente, migrando para o mar para completar seu ciclo, com duração de aproximadamente dois anos (Dall et al., 1990; Pérez-Farfante, 1970). Adultos do camarão branco são encontrados até $50 \mathrm{~m}$ de profundidade, onde se reproduzem (D'Incao, 1995).

Estudos referentes à dinâmica e estrutura populacional do $L$. schmitti foram realizados na região nordeste (Calazans, 2013; Luvesuto, 2006; Peixoto et al., 2018; Santos and Freitas, 
Freire et al., Estrutura populacional e morfometria do camarão branco Litopenaeus schmitti (Burkenroad, 1936) capturado pela pesca artesanal em Sergipe, Brasil.

2004; Santos, 2010; Silva et al., 2018) e sudeste (Barioto, 2017; Carvalho, 2013; Gonçalves et al., 2009; Lopes, 2012; Santos, 2007) e tiveram como finalidade determinar a proporção sexual, relação entre comprimento total e da carapaça, relação peso-comprimento, tamanho de primeira maturação, período reprodutivo, abundância, crescimento, longevidade e mortalidade. Porém, para o estado de Sergipe, somente foram encontrados trabalhos para L. schmitti realizados nos meses de setembro de 2013 a agosto de 2014 (Santos, 2016; Silva, 2016), sendo realizados a partir de dados independentes da pesca. Faz-se necessário uma melhor compreensão da estrutura e dinâmica populacional dessa espécie no estado, em função da sua elevada contribuição econômica. Este estudo tem o objetivo de analisar a estrutura populacional do Litopenaeus schmitti em Sergipe e a sua morfometria, utilizando dados dependentes da pesca que possibilitem um acompanhamento contínuo de baixo custo, e a longo prazo, dos possíveis efeitos da pesca e de outros fatores sobre o estoque local.

\section{MATERIAIS E MÉTODOS}

\section{AMOSTRAGEM DO MATERIAL BIOLÓGICO}

No terminal pesqueiro, foi observado que os pescadores fazem a separação dos camarões em três categorias de tamanho: espigão (camarão pequeno), escolha (camarão médio) e pistola (camarão grande). Porém, somente nas duas últimas categorias estão incluídos espécimes de $L$. schmitti, as quais foram amostradas neste trabalho. Assim, para realizar os estudos referentes à estrutura populacional e morfometria, foram coletados $3 \mathrm{~kg}$ de escolha e $3 \mathrm{~kg}$ de pistola por mês, no período de janeiro de 2015 a março de 2016, no Terminal Pesqueiro do Município de Aracaju, no estado de Sergipe. Nenhuma amostra foi obtida durante os períodos de defeso de $1^{0}$ de abril a 15 de maio e $1^{\circ}$ de dezembro a 15 de janeiro, estabelecidos de acordo com a Instrução Normativa no14 de 14 de outubro de 2004 (MMA, 2004). Essas amostras foram obtidas de embarcações camaroneiras artesanais que atuam ao longo da costa do estado (Fig. $1)$.

\section{ESTRUTURA POPULACIONAL E MORFOMETRIA}

O material coletado foi mantido congelado em freezer no Laboratório de Ecologia Pesqueira do Departamento de Engenharia de Pesca e Aquicultura da Universidade Federal de Sergipe (LEP/DEPAQ/UFS). Em seguida, as amostras foram triadas e todos os exemplares de $L$. schmitti separados das demais espécies de camarão para posterior análise. Foram medidos o comprimento total ( $\mathrm{CT}$, distância entre a extremidade do rostro e a extremidade do télson) e o comprimento da carapaça (CC, desde a base orbital até o final da carapaça), com o auxílio de um paquímetro digital (precisão de $0,01 \mathrm{~mm}$ ), e determinado o peso úmido total (PT), medido com o auxílio de uma balança analítica (precisão de 0,0001 g). Cada indivíduo foi sexado, sendo 
Freire et al., Estrutura populacional e morfometria do camarão branco Litopenaeus schmitti (Burkenroad, 1936) capturado pela pesca artesanal em Sergipe, Brasil.

posteriormente identificado o seu estágio de maturação gonadal. As fêmeas foram identificadas pela presença do télico e o seu estágio de maturação foi avaliado em função da coloração das gônadas (Lara, 1972): estágio I (imatura), estágio II (em desenvolvimento), estágio III (madura) e estágio IV (vazia). Os machos foram avaliados por observação macroscópica do petasma, sendo os não fusionados considerados imaturos e os fusionados, maturos (Amado, 1978).

O teste do qui-quadrado, com a correção de Yates, foi aplicado para testar se a proporção sexual diferia estatisticamente de 1:1 (Zar, 2010). As médias do comprimento da carapaça (CC) e do peso total (PT) dos machos e das fêmeas foram comparadas através de um teste $t$ com variâncias diferentes. Foram ajustadas regressões lineares $(C T=a+b \cdot C C)$ entre o comprimento total e o comprimento da carapaça para machos e fêmeas, separadamente. Para a relação peso-comprimento, foi utilizado o modelo potencial $\left(\mathrm{PT}=\mathrm{a} \cdot \mathrm{C} \mathrm{C}^{\mathrm{b}}\right.$ ) para machos e fêmeas, separadamente, avaliando-se a existência de isometria $(b=3)$, alometria negativa $(b<3)$ ou alometria positiva ( $b>3$ ). A análise de covariância foi aplicada para testar se houve diferença significativa entre machos e fêmeas nas inclinações e nos interceptos das relações comprimento-comprimento e peso-comprimento linearizadas (Zar, 2010). Todos os testes foram realizados com um nível de significância de 5\%, sendo utilizado o Programa R ( $\mathrm{R}$ Core Team, 2018).

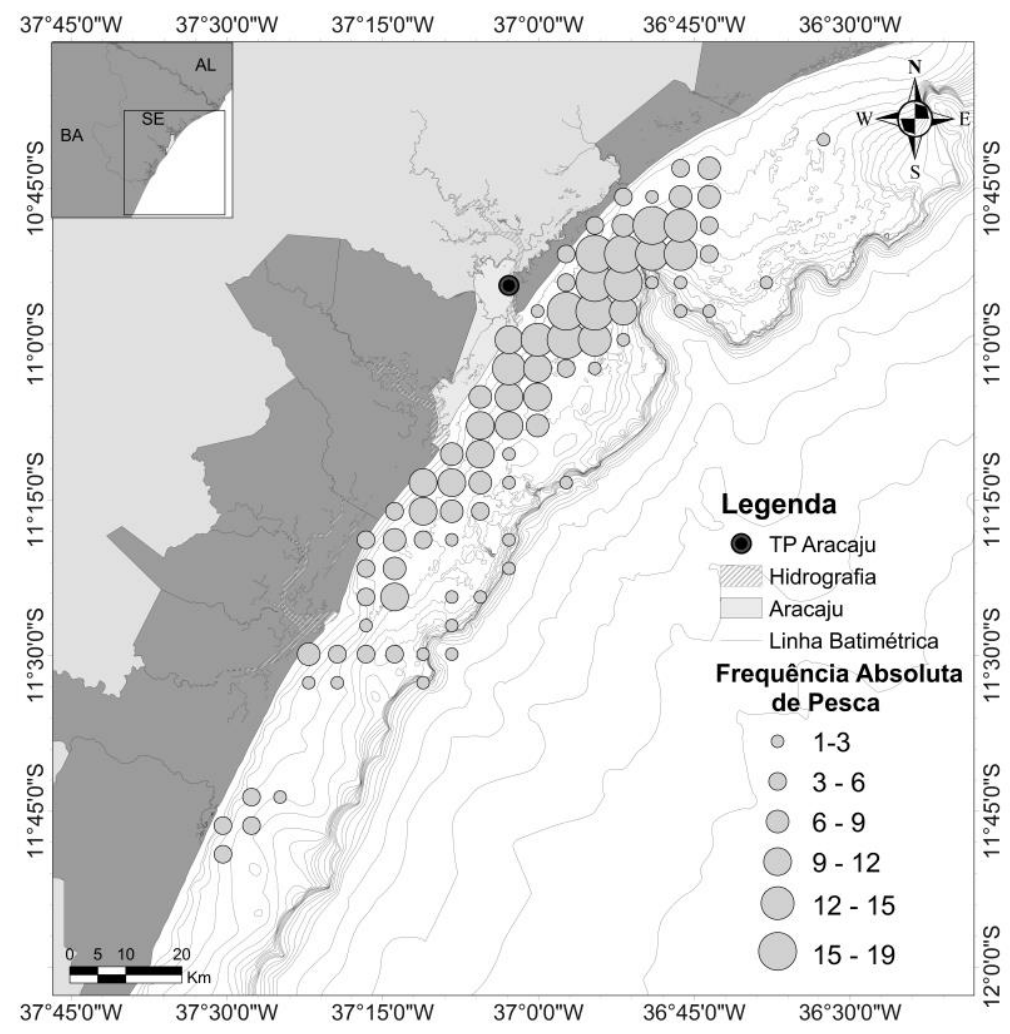

Figura 1. Área de atuação da frota camaroneira de Sergipe. TP = Terminal Pesqueiro (Elaborado por Robson Andrade Rosa, Projeto de Monitoramento Participativo do Desembarque Pesqueiro). 
Freire et al., Estrutura populacional e morfometria do camarão branco Litopenaeus schmitti (Burkenroad, 1936) capturado pela pesca artesanal em Sergipe, Brasil.

\section{RESULTADOS}

As maiores capturas foram observadas nos meses de janeiro de 2015 e 2016 e nenhum indivíduo foi capturado no mês de fevereiro de 2015 (Fig. 2). Desses indivíduos, 81 eram machos e 110 fêmeas, resultando em uma proporção sexual geral de 0,7:1, indicando um predomínio significante de fêmeas $\left(\chi^{2}=4,13 ; p=0,04\right)$. A proporção sexual mensal apresentou diferença significativa somente nos meses de janeiro de 2015 e março de 2016 (Fig. 2).

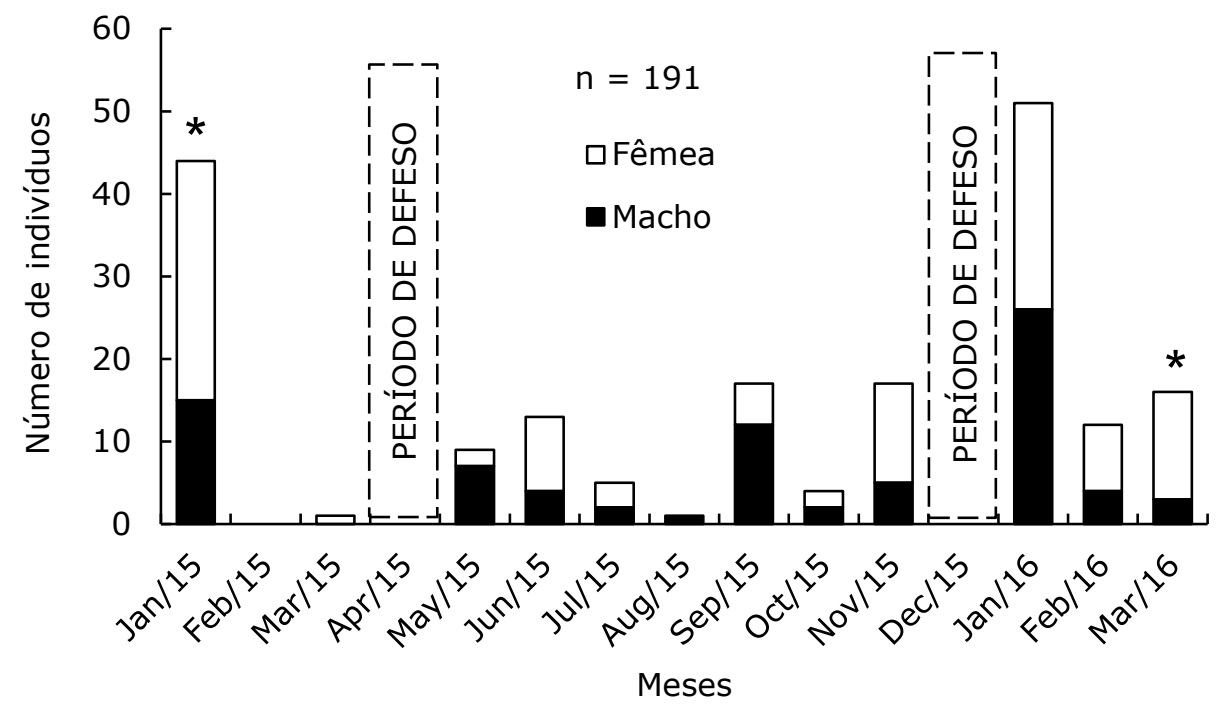

Figura 2. Proporção sexual mensal de Litopenaeus schmitti capturado entre janeiro de 2015 e março de 2016 no litoral de Sergipe, com indicação dos dois períodos de defeso ( $1^{\circ}$ de abril a 15 de maio e $1^{\circ}$ de dezembro a 15 de janeiro). Asteriscos representam diferenças significativas $(p<0,05)$.

O comprimento da carapaça (CC) dos machos variou de 21,69 a $35,16 \mathrm{~mm}(26,85 \pm 2,73$

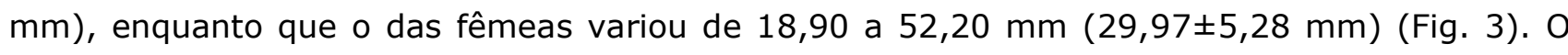
teste $t$ indicou que o CC médio das fêmeas é estatisticamente superior ao dos machos ( $t=5,19$; $\mathrm{p}<0,001)$.

Não foi possível estimar o tamanho de primeira maturação nem o período reprodutivo devido ao reduzido número de indivíduos presentes nas amostras coletadas. A quantidade de indivíduos por estágio de desenvolvimento gonadal está descrita na Tabela 1.

A relação entre o comprimento total (CT) e o comprimento da carapaça (CC) estimada para machos foi $C T=25,960+3,837 C C$ e para fêmeas foi $C T=33,199+3,502 C C$ (Fig. 4A, B). A análise de covariância não indicou diferença significativa na inclinação $(p=0,28)$, mas sim nos interceptos $(p=0,01)$.

O peso total (PT) dos machos variou de 8,33 a 27,88 $\mathrm{g}(16,39 \pm 4,34 \mathrm{~g})$ e o das fêmeas de 4,18 a $64,00 \mathrm{~g}(20,55 \pm 9,51 \mathrm{~g})$. O peso médio das fêmeas foi significativamente superior ao dos 
Freire et al., Estrutura populacional e morfometria do camarão branco Litopenaeus schmitti (Burkenroad, 1936) capturado pela pesca artesanal em Sergipe, Brasil.

machos $(t=3,96 ; p<0,001)$. A relação entre o peso total e o comprimento da carapaça estimada para os machos foi $\mathrm{PT}=0,00303 \mathrm{CC}^{2,609}$ e para as fêmeas foi $\mathrm{PT}=0,00248 C C^{2,642}$ (Fig. 5A, B). A análise de covariância para os modelos potenciais linearizados dos machos e das fêmeas não indicou diferença significativa nas declividades $(p=0,63)$, mas sim nos interceptos $(p<0,01)$.

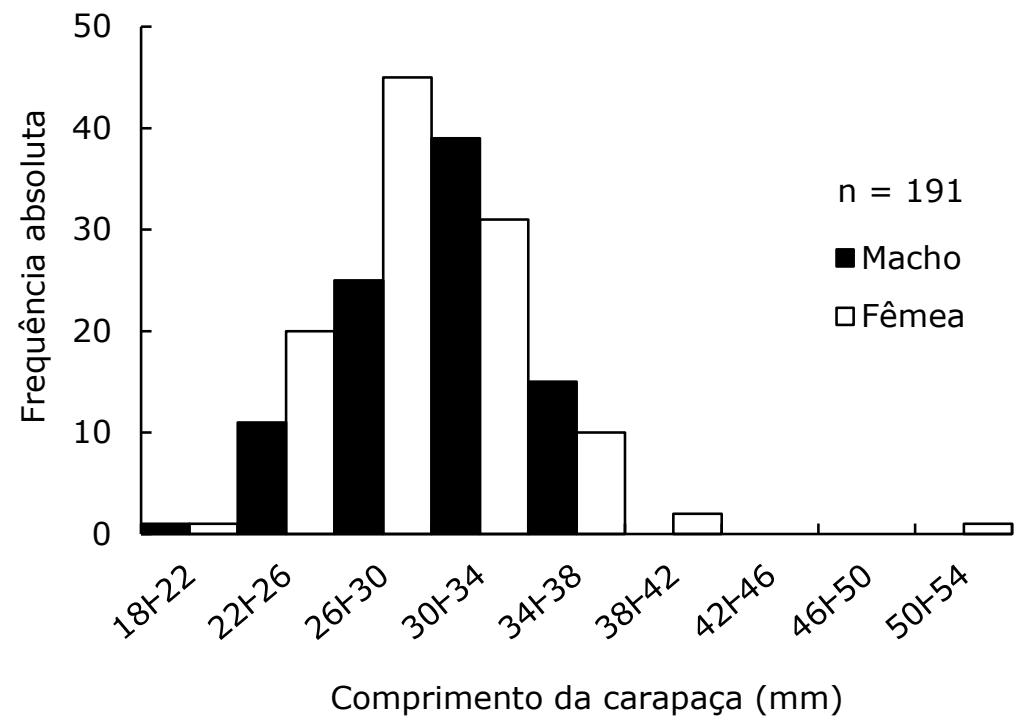

Figura 3. Distribuição de frequência de comprimento da carapaça para machos e fêmeas de Litopenaeus schmitti capturados mensalmente entre janeiro de 2015 e março de 2016 no litoral de Sergipe.

Tabela 1. Número de indivíduos de Litopenaeus schmitti por estágio de maturação gonadal coletados na costa de Sergipe entre janeiro de 2015 e março de 2016.

\begin{tabular}{lcc}
\hline \multicolumn{1}{c}{ Sexo } & Estágio de maturação & Frequência relativa (\%) \\
\hline Macho & & \\
Imaturo & 4 & 2,1 \\
$\quad$ Maturo & 77 & 40,3 \\
Fêmea & 14 & 7,3 \\
$\quad$ Imatura & 29 & 15,2 \\
Em desenvolvimento & 3 & 1,6 \\
Madura & 64 & 33,5 \\
$\quad$ Vazia & 191 & 100,0 \\
Total & &
\end{tabular}


Freire et al., Estrutura populacional e morfometria do camarão branco Litopenaeus schmitti (Burkenroad, 1936) capturado pela pesca artesanal em Sergipe, Brasil.
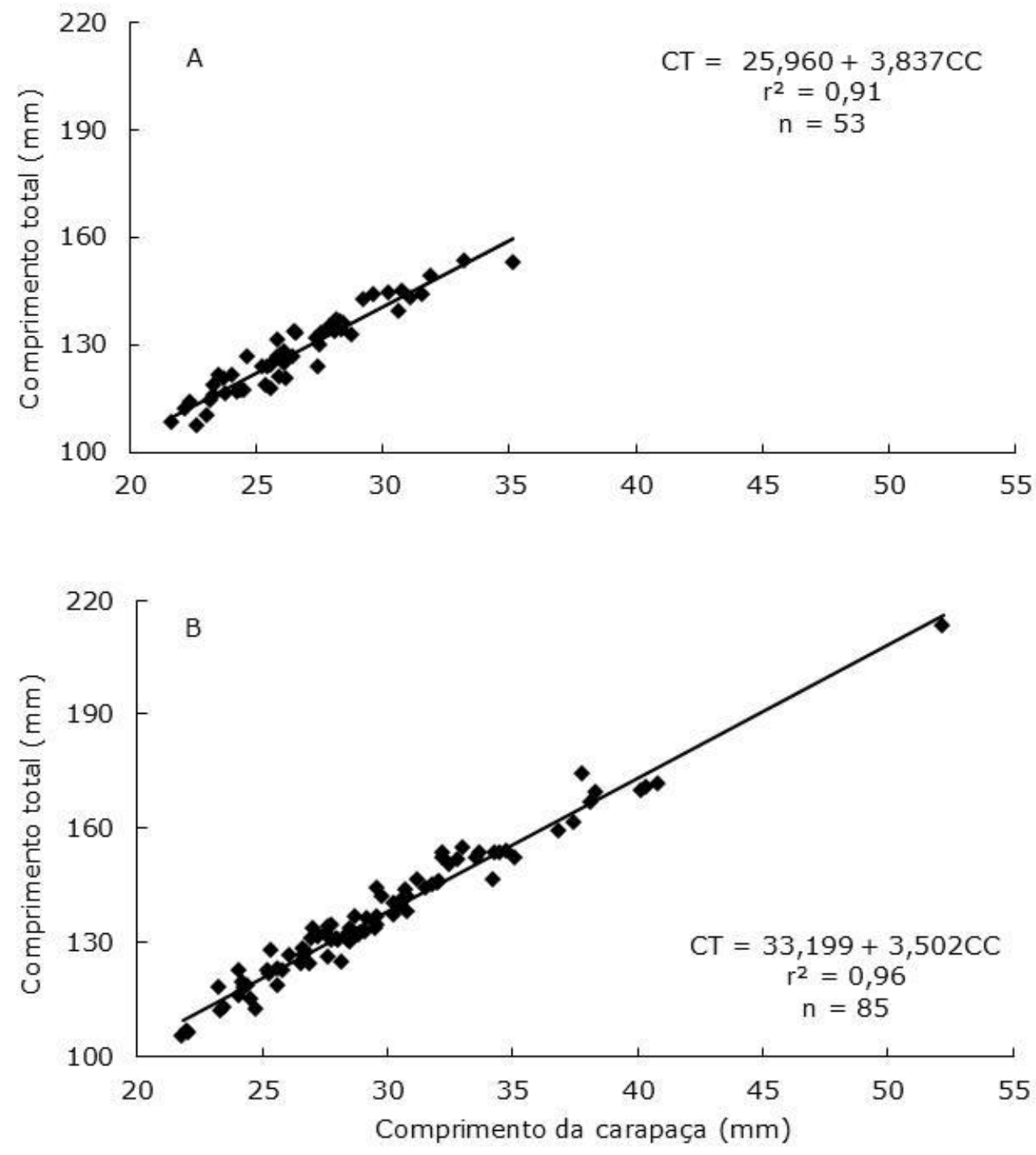

Figura 4. Relação entre o comprimento total (CT) e o comprimento da carapaça (CC) para machos (A) e fêmeas (B) de Litopenaeus schmitti capturados entre janeiro de 2015 e março de 2016 no litoral de Sergipe. 
Freire et al., Estrutura populacional e morfometria do camarão branco Litopenaeus schmitti (Burkenroad, 1936) capturado pela pesca artesanal em Sergipe, Brasil.
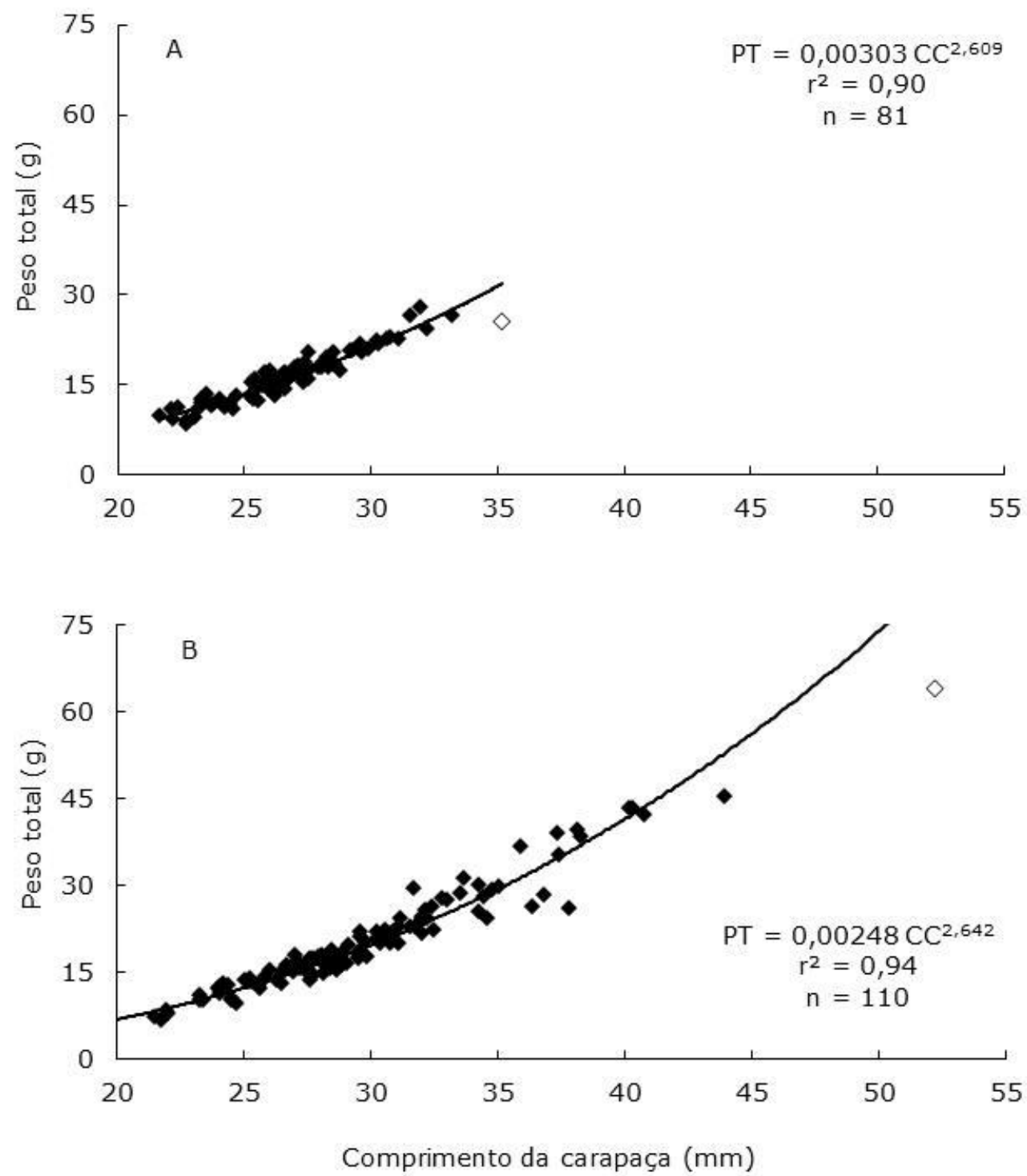

Figura 5. Relação entre o peso total (PT) e o comprimento da carapaça (CC) para machos (A) e fêmeas (B) de Litopenaeus schmitti capturados entre janeiro de 2015 e março de 2016 no litoral de Sergipe. Os losangos brancos indicam pontos discrepantes, os quais foram excluídos das relações estimadas. 
Freire et al., Estrutura populacional e morfometria do camarão branco Litopenaeus schmitti (Burkenroad, 1936) capturado pela pesca artesanal em Sergipe, Brasil.

\section{DISCUSSÃO}

Neste estudo foi verificado que houve menor número de machos em relação ao de fêmeas de L. schmitti, mesmo padrão já observado em Sergipe (Silva, 2016; Santos, 2016), assim como em várias localidades no litoral brasileiro (Tab. 2). Maior proporção relativa de fêmeas nas populações de camarões peneídeos é um fato comum, o que pode ser associado a altas taxas de mortalidades nos machos (Cha et al., 2002). Adicionalmente, ao longo da maturação gonadal, as fêmeas passam maiores períodos de tempo alimentando-se próximo ao substrato para suprirem altas taxas de metabolismo, ficando mais vulneráveis à captura pelas artes de pesca (Keyrekidis and Thessalou-Legaki, 2006). Os machos são menos vulneráveis à captura devido ao comportamento de se enterrar mais do que as fêmeas no sedimento ou permanecer por maior tempo na coluna d'água (Santos and Freitas, 2004).

Tabela 2. Número de machos e fêmeas de Litopenaeus schmitti e proporção sexual de acordo com diferentes estudos realizados na costa do Brasil. *Indica proporção sexual estatisticamente diferente de $1: 1$.

\begin{tabular}{lcccc}
\hline \multicolumn{1}{c}{ Localidade/Estado } & $\begin{array}{c}\text { Número de } \\
\text { machos }\end{array}$ & $\begin{array}{c}\text { Número de } \\
\text { fêmeas }\end{array}$ & $\begin{array}{c}\text { Proporção } \\
\text { sexual }\end{array}$ & Referência \\
\hline Pernambuco & 491 & 678 & $0,7: 1^{*}$ & Peixoto et al. (2018) \\
Coruripe/Alagoas & 851 & 792 & $1,1: 1$ & Santos (2010) \\
Sergipe & 208 & 296 & $0,7: 1$ & Silva (2016) \\
Sergipe & 81 & 110 & $0,7: 1^{*}$ & Presente estudo \\
Rio de Janeiro/Rio de Janeiro & 437 & 355 & $1,2: 1$ & Carvalho (2013) \\
Baixada Santista/São Paulo (mar) & 440 & 1130 & $0,4: 1^{*}$ & Santos et al. (2008) \\
Baixada Santista/São Paulo (estuário) & 334 & 1008 & $0,3: 1^{*}$ & Santos et al. (2008) \\
Estuário de São Vicente/São Paulo & 800 & 1041 & $0,8: 1^{*}$ & Lopes (2012) \\
Baía de São Vicente/São Paulo & 1502 & 1772 & $0,9: 1^{*}$ & Lopes (2012) \\
Cananéia/São Paulo & 504 & 598 & $0,8: 1^{*}$ & Barioto (2017) \\
\hline
\end{tabular}

A análise da proporção sexual por mês foi prejudicada pelo reduzido número de exemplares obtidos nas amostras, embora todos os indivíduos dessa espécie presentes nas amostras tenham sido incluídos nas análises (Tab. 1). Uma vez que a categoria pistola é geralmente associada a L. schmitti (Araújo et al., 2016; Thomé-Souza et al., 2014), assim como o camarão grande (Dias-Neto, 2011), não se esperava obter um tamanho amostral tão reduzido ao se considerar todos os exemplares da categoria pistola em conjunto com a categoria escolha. Dados de levantamentos anteriores têm demonstrado a baixa proporção ( $<7 \%)$ de L. schmitti em relação ao total de camarões capturados na costa de Sergipe (Santos et al., 2007; Santos et al., 2017; Silva, 2016). Porém, a razão para tal baixa abundância permanece ainda desconhecida.

As maiores capturas de $L$. schmitti ocorreram no período do verão, logo após o defeso no final do ano, sendo similar ao encontrado por Santos et al. (2017) para o estado de Sergipe e 
Freire et al., Estrutura populacional e morfometria do camarão branco Litopenaeus schmitti (Burkenroad, 1936) capturado pela pesca artesanal em Sergipe, Brasil.

por Santos (2007) para o estado de São Paulo. Este fator pode ser explicado pelo período de defeso em que as frotas camaroneiras não estão em atividade devido ao período reprodutivo dos camarões, conforme a Instrução Normativa n¹4 de 14 de outubro de 2004 (MMA, 2004). Apesar de não ter sido possível estimar o tamanho de primeira maturação (devido ao reduzido número de exemplares, Tab. 1), pode-se considerar os dados de Silva (2016) para a mesma região. De acordo com a autora, o comprimento de primeira maturação para os machos é de $18,3 \mathrm{~mm}$ CC e para as fêmeas é de $19,6 \mathrm{~mm}$ CC. No presente trabalho foi verificado apenas um indivíduo com comprimento da carapaça inferior a $20,00 \mathrm{~mm}$ e, portanto, abaixo do tamanho de primeira maturação $\left(\mathrm{CC}_{\mathrm{m}}\right)$. Entretanto, 9,4\% dos exemplares analisados, dentre machos e fêmeas, estavam imaturos (Tab. 1).

As fêmeas apresentaram um comprimento médio da carapaça superior ao dos machos, indicando um dimorfismo sexual para essa espécie, o qual é regra geral nos camarões peneídeos (Boschi, 1969). Este padrão também foi encontrado para a espécie em estudo por Luvesuto (2006) em três pontos diferentes da costa do Rio Grande do Norte (Baía Formosa, Touros e Diogo Lopes), por Silva et al. (2018) em Pernambuco, por Santos (2010) em Coruripe $(A L)$ e por Bochini (2012) na região de Ubatuba (SP). Em outras espécies de peneídeos, o dimorfismo sexual também é característico: Reis Jr. et al. (2017) para X. kroyeri, Costa and Fransozo (1999) para F. brasiliensis e F. paulensis, e Castilho et al. (2008) para P. muelleri. Essa característica está associada à reprodução, uma vez que maiores tamanhos indicam maior produção de ovócitos e, proporcionalmente, uma maior fecundidade esperada (Gab-Alla et al., 1990).

No presente trabalho foi encontrado um comprimento da carapaça (CC) máximo de 52,50 mm, muito superior ao observado por Silva (2016) para Sergipe $(39,30 \mathrm{~mm})$, que capturou os indivíduos apenas na região entre os estuários dos rios Sergipe e Vaza Barris, utilizando barcos comerciais contratados, com as mesmas características utilizadas nesse trabalho e com a mesma arte de pesca. Porém, como as amostras utilizadas aqui não foram georeferenciadas, não se sabe ao certo a sua procedência precisa ao longo da costa. Essa diferença de valor máximo observado pode afetar estimativas de curvas de crescimento para camarões utilizando distribuições de frequência de comprimento. A amplitude do comprimento da carapaça (CC) neste estudo foi bem próxima à obtida no trabalho de Santos (2010) em Coruripe (no estado de Alagoas) e no de Bochini (2012) na região de Ubatuba (no estado de São Paulo), indicando que Silva (2016) pode ter subestimado o valor do comprimento assintótico.

Do mesmo modo que o comprimento, o peso médio dos machos $(16,34 \mathrm{~g})$ também foi inferior ao das fêmeas $(20,45 \mathrm{~g})$. Essa tendência é comum em $L$. schmitti, como observado por Luvesuto (2006) no Rio Grande do Norte e em outros peneídeos, já que as fêmeas necessitam atingir tamanhos maiores para reprodução, como previamente discutido (ver Tonial (2011) para X. kroyeri na costa de Sergipe e Aragão (2012) para F. subtilis ao longo do litoral brasileiro). Isso também está refletido nas relações comprimento total-comprimento da carapaça estimadas 
Freire et al., Estrutura populacional e morfometria do camarão branco Litopenaeus schmitti (Burkenroad, 1936) capturado pela pesca artesanal em Sergipe, Brasil.

para machos e fêmeas, em que a inclinação da reta não é estatisticamente diferente, indicando um crescimento proporcional semelhante, porém a partir de tamanhos maiores nas fêmeas, evidenciado pela diferença no intercepto dessa mesma relação. Esse mesmo padrão foi observado na relação peso-comprimento de machos e fêmeas, com uma alometria negativa $(b<3)$ observada, como preponderantemente encontrada ao longo da costa brasileira (Tab. 3).

Tabela 3. Relação entre peso total e comprimento da carapaça $\left(P T=a \cdot C C^{b}\right)$ para machos e fêmeas de Litopenaeus schmitti em distintos estados do Brasil. $a=$ intercepto, $b=$ inclinação, $r^{2}=$ coeficiente de determinação, $\mathrm{n}=$ número de indivíduos amostrados e $\mathrm{CC}=$ comprimento mínimo e máximo da carapaça.

\begin{tabular}{|c|c|c|c|c|c|c|c|}
\hline $\begin{array}{l}\text { Localidade/ } \\
\text { Estado }\end{array}$ & Sexo & $a$ & $b$ & $r^{2}$ & $n$ & $\mathrm{CC}(\mathrm{mm})$ & Referência \\
\hline \multirow{2}{*}{$\begin{array}{l}\text { Diogo Lopes/ } \\
\text { Rio Grande do Norte }\end{array}$} & Macho & 0,0012 & 2,9043 & 0,95 & 33 & $23,70-34,00$ & \multirow{2}{*}{$\begin{array}{l}\text { Luvesuto } \\
\text { (2006) }\end{array}$} \\
\hline & Fêmea & 0,0057 & 2,4321 & 0,93 & 21 & $27,60-44,80$ & \\
\hline \multirow[t]{2}{*}{$\begin{array}{l}\text { Touros/ } \\
\text { Rio Grande do Norte }\end{array}$} & Macho & 0,0070 & 3,0181 & 0,95 & 34 & $25,00-37,70$ & \multirow[t]{2}{*}{$\begin{array}{l}\text { Luvesuto } \\
\text { (2006) }\end{array}$} \\
\hline & Fêmea & 0,0020 & 2,6884 & 0,97 & 18 & $18,30-53,80$ & \\
\hline \multirow[t]{2}{*}{$\begin{array}{l}\text { Baía Formosa/ } \\
\text { Rio Grande do Norte }\end{array}$} & Macho & 0,0040 & 2,5026 & 0,83 & 32 & $24,00-35,80$ & \multirow[t]{2}{*}{$\begin{array}{l}\text { Luvesuto } \\
\text { (2006) }\end{array}$} \\
\hline & Fêmea & 0,0053 & 2,4095 & 0,94 & 18 & $27,60-48,40$ & \\
\hline \multirow[t]{2}{*}{ Sergipe } & Macho & 0,0030 & 2,6090 & 0,90 & 81 & $21,69-35,16$ & \multirow[t]{2}{*}{$\begin{array}{l}\text { Presente } \\
\text { estudo }\end{array}$} \\
\hline & Fêmea & 0,0030 & 2,6420 & 0,94 & 110 & $18,90-52,50$ & \\
\hline \multirow[t]{2}{*}{$\begin{array}{l}\text { Rio de Janeiro/Rio } \\
\text { de Janeiro }\end{array}$} & Macho & 0,0051 & 3,1500 & 0,96 & 437 & $11,40-37,92$ & \multirow[t]{2}{*}{$\begin{array}{l}\text { Carvalho } \\
(2013)\end{array}$} \\
\hline & Fêmea & 0,0077 & 2,9900 & 0,97 & 355 & $9,40-53,48$ & \\
\hline \multirow{2}{*}{$\begin{array}{l}\text { Baixada Santista/ } \\
\text { São Paulo }\end{array}$} & Macho & 0,0252 & 2,0130 & 0,68 & 774 & $15,00-29,00$ & \multirow[t]{2}{*}{ Santos (2007) } \\
\hline & Fêmea & 0,0114 & 2,2378 & 0,87 & 2138 & $10,00-35,00$ & \\
\hline
\end{tabular}

\section{AGRADECIMENTOS}

Agradecemos a Josafá Reis Jr., Thaíza Barreto e Aline Dias pelo auxílio no processamento das amostras; ao coletor Diógenis Lopes e aos mestres de embarcações camaroneiras por auxiliarem na obtenção das amostras no Terminal Pesqueiro de Aracaju; ao Projeto de Monitoramento Participativo de Desembarque Pesqueiro (PMPDP) e à Fundação de Apoio à Pesquisa e Extensão de Sergipe (FAPESE) pelas bolsas de iniciação científica; e a Jodnes Sobreira Vieira pelos comentários e sugestões. 
Freire et al., Estrutura populacional e morfometria do camarão branco Litopenaeus schmitti (Burkenroad, 1936) capturado pela pesca artesanal em Sergipe, Brasil.

\section{REFERÊNCIAS}

Amado, M.A.P.M. (1978), Estudos biológicos do Xiphopenaeus kroyeri (Heller, 1862), camarão sete-barbas (Crustacea, Penaeidae), de Matinhos, Paraná, Dissertação de Mestrado, Universidade Federal do Paraná, 94p.

Aragão, J.A.N. (2012), Dinâmica populacional e avaliação de estoque do camarão rosa Farfantepenaeus subtilis (Pérez-Farfante, 1967) na plataforma continental amazônica brasileira, Tese de Doutorado, Escola de Engenharia de São Carlos, Universidade de São Paulo, 245p.

Araújo, A.R.R., Barbosa, J.M., Santos, J.P., Carvalho, B.L.F., Garciov-Filho, E.B., Deda, M.S., Silva, C.O. and Chammas, M.A. (2016), Boletim estatístico da pesca nos litorais de Sergipe e extremo norte da Bahia - 2014. Editora UFS, São Cristóvão, 84p.

Barioto, J.G. (2017), Ecologia e estrutura populacional do camarão-branco Litopenaeus schmitti (Burkenroad, 1936) (Dendrobranchiata: Penaeoidea) na região de Cananéia, litoral sul do estado de São Paulo, Dissertação de Mestrado, Universidade Estadual Paulista "Júlio de Mesquita Filho" - UNESP, Pós-Graduação em Ciências Biológicas - AC: Zoologia, 76p.

Bate, C.S. (1888), Report on the Crustacea Macrura collected by H. M. S. challenger during the years 1873-76. Report of the Scientific Results of the Voyage of the Challenger (Zoology), Vol. 24, pp. 1-942.

Bochini, G.L. (2012), Distribuição ecológica e estrutura populacional em escala espacial, temporal e anual do camarão-branco Litopenaeus schmitti (Burkenroad, 1936) (Dendrobranchiata: Penaeidae) na enseada de Ubatuba: 4 anos de estudos, Dissertação de Mestrado, Pós-Graduação em Ciências Biológicas, Universidade Estadual Paulista, 85p.

Boschi, E.E. (1969), Estudio biologico pesquero del camaron Artemesia longinaris Bate de Mar del Plata, Boletín del Instituto de Biologia Marina Mar del Plata, Vol. 18, pp. 1- 47. DOI?

Burkenroad, M.D. (1936), A new species of Penaeus from the American Atlantic. Anais de Academia Brasileira de Ciências, Vol. 8, pp. 315-318.

Calazans, N.K.F. (2013), Dinâmica reprodutiva do camarão branco Litopenaeus schmitti (Burkenroad, 1936) (Decapoda: Penaeidae) no litoral de Pernambuco, Dissertação de Mestrado, Universidade Federal Rural de Pernambuco, Programa de Pós-Graduação em Recursos Pesqueiros e Aquicultura, 70p.

Carvalho, C. (2013), Crescimento e mortalidade do camarão branco Litopenaeus schmitti (Burkenroad, 1936) (Crustacea: Decapoda: Penaeidae) em ambiente natural e em confinamento, Dissertação de Mestrado, Programa de Pós-Graduação em Biologia Animal, Universidade Federal Rural do Rio de Janeiro, 90p. 
Freire et al., Estrutura populacional e morfometria do camarão branco Litopenaeus schmitti (Burkenroad, 1936) capturado pela pesca artesanal em Sergipe, Brasil.

Castilho, A.L., Costa, R.C., Fransozo, A. and Negreiros-Franzoto, M.L. (2008), Reproduction and recruitment of the South American red shrimp, Pleoticus muelleri (Crustacea: Solenoceridae), from the southeastern coast of Brazil, Invertebrate Reproduction \& Development, Vol. 52(1-2), pp. 59-68. DOI: 10.1080/17451000802029536

Cha, H.K., Oh, C., Hong, S.Y. and Park, K.Y. (2002), Reproduction and population dynamics of Penaeus chinensis (Osbeck, 1765) (Decapoda: Penaeidae) on the western coast of Korea, Yellow Sea Fisheries Research, Vol. 56, pp. 25-36. DOI: 10.1016/S0165-7836(01)00310-1

Costa, R.C. and Fransozo, A.A. (1999), Nursery ground for two tropical pink-shrimp Penaeus species: Ubatuba Bay, northern coast of São Paulo, Brazil, Nauplius, Vol. 7, pp. 73-81.

Dall, W., Hill, B.J., Rothlisberg, P.C. and Sharples, D.J. (1990), The biology of Penaeidae, Advances in Marine Biology, Vol. 27, pp. 1-484.

Dias-Neto, J. (2011), Proposta de Plano Nacional de Gestão para o Uso Sustentável de Camarões Marinhos do Brasil, IBAMA, Brasília, 243p.

Diegues, C.A.S. (1983), Pescadores camponeses e trabalhadores do Mar, Série Ensaios, Ática Editora, São Paulo, 292p.

D'Incao, F. (1995), Taxonomia, padrões distribucionais e ecológicos dos Dendrobranchiata (Crustacea, Decapoda) do Brasil e Atlântico Ocidental, Tese de Doutorado, Universidade Federal do Paraná, 365p.

FAO (2016), The state of world fisheries and aquaculture, Contributing to food security and nutrition for all, Food and Agriculture Organization, Rome, 200p.

Flores-Montes, M.J. (2003), Fatores que influenciam na produtividade dos oceanos: a importância do fluxo de difusão dos nutrientes para a biomassa do fitoplâncton na região oceânica do nordeste brasileiro, Tese de Doutorado, Programa de Pós-Graduação em Oceanografia, Universidade Federal de Pernambuco, 198p.

Fundação BIO-RIO (2002), Avaliação e ações prioritárias para a conservação da biodiversidade das zonas costeira e marinha, Secretaria de Biodiversidade e Florestas (SBF), Ministério do Meio Ambiente (MMA), Brasília.

Gab-Alla, A.A., Hartnoll, R.G., Ghobashy, A.F. and Mohammed, S.Z. (1990), Biology of penaeid prawns in the Suez Canal lakes, Marine Biology, Vol. 107(3), pp. 417-426. DOI: 10.1007/BF01313423

Gonçalves, S.M., Santos, J.L. and Rodrigues, E.S. (2009), Estágios de desenvolvimento gonadal de fêmeas do camarão branco Litopenaeus schmitti (Burkenroad, 1936), capturadas na região marinha da Baixada Santista, São Paulo, Revista Ceciliana, Vol. 1(2), pp. 96-100. 
Freire et al., Estrutura populacional e morfometria do camarão branco Litopenaeus schmitti (Burkenroad, 1936) capturado pela pesca artesanal em Sergipe, Brasil.

Haimovici, M., Andriguetto Filho, J.M. and Sunyé, P.S. (2014), A pesca marinha e estuarina no Brasil: estudos de caso multidisciplinares, Editora da FURG, Rio Grande, 192p.

Heller, C. (1862), Beitrage zur naheren Kenntnis der Macrouren. Sitzungsberichte der mathematischnaturwissenschaftlichen Classe der Kaiserlichen Akademie der Wissenschaften in Wien, pp. 389-426.

IBAMA (2007), Estatística da Pesca 2007 (Brasil) - Grandes Regiões e Unidades da Federação, Instituto Brasileiro do Meio Ambiente e dos Recursos Naturais Renováveis, Brasília-DF, $174 p$.

Kevrekidis, K. and Thessalou-Legaki, M. (2006), Catch rates size structure and sex ratio of Melicertus kerathurus (Forskal, 1775) (Decapoda: Penaeidae) from an Aegean Sea trawl fishery, Fisheries Research, Vol. 80, pp. 270-279. DOI: 10.1016/j.fishres.2006.04.001

Lara, M.D.B.G. (1972), Estudos preliminares da maturação da gônada do camarão legítimo Penaeus schmitti, Acarpesc Cientific, Vol. 1, pp. 1-24.

Latreille, P.A. (1817), Penee. Penaeus, Nouveau Dictionnaire d'Histoire Naturelle, Vol. 25, pp. 152-156.

Lopes, M. (2012), Distribuição e dinâmica populacional dos camarões-rosa, Farfantepenaeus brasiliensis (Latreille, 1817) e F. paulensis (Pérez-Farfante, 1967) e do camarão-branco Litopenaeus schmitti (Burkenroad, 1936) (Decapoda: Dendrobranchiata: Penaeidae) no complexo baía-estuário de Santos-São Vicente, São Paulo, Brasil: Subsídios científicos para a averiguação do período ideal de defeso, Tese de Doutorado, Instituto de Biociências, Universidade Estadual Paulista "Júlio Mesquita Filho", 163p.

Luvesuto, E. (2006), Análise genética e morfométrica da estrutura populacional do camarão branco Litopenaeus schmitti (Decapoda, Crustacea) na costa do Rio Grande do Norte, Brasil: uma abordagem em fina escala, Dissertação de Mestrado, Programa de PósGraduação em Ecologia e Recursos Naturais, Universidade Federal de São Carlos, 91p.

MMA (2004), Instrução Normativa No14, de 14 de outubro de 2004. Ministério do Meio Ambiente. Proibição anualmente do exercício da pesca de camarão rosa camarão setebarbas e camarão branco, com quaisquer artes de pesca, nas áreas compreendidas entre a divisa dos Estados de Pernambuco e Alagoas e a divisa dos Municípios de Mata de São João e Camaçari no Estado da Bahia, nos períodos de $1^{\circ}$ de abril a 15 de maio e $1^{\circ}$ de dezembro a 15 de janeiro. Brasília - DF.

Peixoto, S., Calazans, N., Silva, E.F., Nole, L., Soares, R. and Frédou, F.L. (2018), Reproductive cycle and size at first sexual maturity of the white shrimp Penaeus schmitti (BURKENROAD, 1936) in northeastern Brazil, Latin American Journal of Aquatic Research, Vol. 46, n. 1, pp. 1-9. DOI: 10.3856/vol46-issue1-fulltext-1 
Freire et al., Estrutura populacional e morfometria do camarão branco Litopenaeus schmitti (Burkenroad, 1936) capturado pela pesca artesanal em Sergipe, Brasil.

Pérez Farfante, I. (1967), A new species and two new subspecies of shrimp of the genus Penaeus from the Western Atlantic. Proceedings of the Biological Society of Washington, Vol. 80, pp. 83-100.

Pérez-Farfante, I. (1970), Sinopsis de datos biológicos sobre el camarón blanco Penaeus schmitti (Burkenroad, 1936), FAO Fisheries and Aquaculture Report, Vol. 57, pp. 14171438.

Pérez-Farfante, I. and Kensley B. (1997), Penaeids and sergestoid shrimps and prawns of the world, Keys and diagnoses for the families and genera, Museum National d'Histoire Naturelle, Paris, 233p.

R Core Team (2018). R: A language and environment for statistical computing. R Foundation for Statistical Computing, Vienna, Austria. https://www.r-project.org/.

Reis Jr. J.J.C., Freire, K.M.F., Rosa, L.C., Barreto, T.M.R.R. and Pauly, D. (2017), Population dynamics of Atlantic seabob Xiphopenaeus kroyeri (Decapoda: Penaeidae) off the state of Sergipe, north-eastern Brazil, Journal of the Marine Biological Association of the United Kingdom, pp. 1-11. DOI: 10.1017/S0025315417001916

Santos, J.L. (2007), Pesca e estrutura populacional do camarão branco Litopenaeus schmitti (Burkenroad, 1936) na região marinha e estuarina da Baixada Santista, São Paulo, Brasil, Dissertação de Mestrado, Programa de Pós-Graduação em Aquicultura e Pesca, Instituto de Pesca, 117p.

Santos, J.L., Severino-Rodrigues, E. and Vaz-dos-Santos, A.M. (2008), Estrutura populacional do camarão branco Litopenaeus schmitti nas regiões estuarina e marinha da Baixada Santista, São Paulo, Brasil, Boletim do Instituto de Pesca, Vol. 34(3), pp. 375-389.

Santos, M.C.F. (2010), Informações biológicas e pesqueiras sobre o camarão branco Litopenaeus schmitti (BURKENROAD, 1936) e o camarão rosa Farfantepenaeus subtilis (PÉREZ-FARFANTE, 1967) capturados no município de Coruripe (Alagoas-Brasil), Boletim Técnico-Científico do CEPENE, Vol. 18(1), pp. 17-29.

Santos, M.C.F. and Freitas, A.E.T.S. (2004), Estrutura populacional e pesca do camarão branco, Penaeus schmitti (Burkenroad, 1936) (Crustacea, Decapoda, Penaeidae) na lagoa Papari, município de Nísia Floresta (Rio Grande do Norte - Brasil), Boletim Técnico-Científico do CEPENE, Vol. 12(1), pp. 23-42.

Santos, M.C.F., Silva, A.C.C.D., Freitas, A.E.T.S. and Sousa, G.S. (2007), Prospecção de camarões marinhos (Crustacea, Decapoda, Penaeidae) na plataforma continental do Estado de Sergipe - Brasil. Boletim Técnico-Científico do CEPENE, Vol. 15(2), pp. 47-56.

Santos, R.C. (2016), Distribuição espaço-temporal de camarões peneídeos (Crustacea; Decapoda: Dendrobranchiata) na plataforma continental de Sergipe, Dissertação de 
Freire et al., Estrutura populacional e morfometria do camarão branco Litopenaeus schmitti (Burkenroad, 1936) capturado pela pesca artesanal em Sergipe, Brasil.

Mestrado, Programa de Pós-Graduação em Ecologia e Conservação, Universidade Federal de Sergipe, 81p.

Santos, R.C., Silva, S.L.R., Costa, R.C., Davanso, T.M. and Hirose, G.L. (2017), Evaluation of the management plan for penaeid shrimps in the continental shelf of Sergipe, Brazil, Boletim do Instituto de Pesca, Vol. 43, n. 3, pp. 308-321. DOI: 10.20950/16782305.2017v43n3p308

Silva, E.F., Calazans, N., Nolé, L., Soares, R., Frédou, F.L. and Peixoto, S. (2018), Population dynamics of the white shrimp Litopenaeus schmitti (Burkenroad, 1936) on the southern coast of Pernambuco, north-eastern Brazil, Journal of the Marine Biological Association of the United Kingdom, pp. 1-7. DOI: 10.1017/S0025315418000322

Silva, S.L.R. (2016), Biologia reprodutiva e crescimento de camarões marinhos (Decapoda: Penaeidae) na plataforma continental de Sergipe, Dissertação de Mestrado, Programa de Pós-Graduação em Ecologia e Conservação, Universidade Federal de Sergipe, 111p.

Thomé-Souza, M.J.F., Carvalho, B.L.F., Garciov-Filho, E.B., Silva, C.O., Deda, M.S., Félix, D.C.F. and Santos, J.C. (2014), Estatística Pesqueira da Costa do Estado de Sergipe e Extremo Norte da Bahia 2013, Editora UFS, São Cristóvão, 101p.

Tonial, L.S.S. (2011), Estrutura populacional do camarão sete barbas Xiphopenaeus kroyeri (Heller, 1862) na foz do rio São Francisco, nordeste do Brasil, Dissertação de Mestrado, Programa de Pós-Graduação em Diversidade Biológica e Conservação nos Trópicos, Universidade Federal de Alagoas, 67p.

Vasconcellos, M., Diegues, A.C. and Sales, R.R. (2007), Limites e possibilidades na gestão da pesca artesanal costeira, In: Costa, A. (org.), Nas redes da pesca artesanal, Brasília: IBAMA, PNUD, 308p.

Zar, J.H. (2010), Biostatistical Analysis, 5a edição, Pearson Prentice Hall, 960p. 